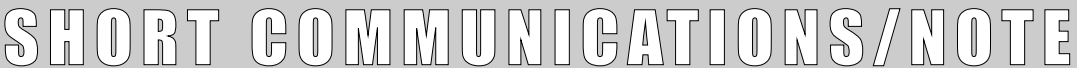

\section{Dientamoeba fragilis nella popolazione suina: indagini preliminari}

\author{
Daniele Crotti', , Marco Sensi', Elisabetta Manuali', Silvia Crotti' \\ 'Istituto Zooprofilattico Sperimentale dell'Umbria e delle Marche,Via G. Salvemini, I - 06I26 Perugia \\ ${ }^{2}$ L. P. in Parassitologia e Microbiologia Medica, S. C. per Pilonico Paterno, 4 - Pianello, Perugia
}

Keywords: Dientamoeba fragilis, diagnosis, epidemiology, Giemsa stain, swine

Dientamoeba fragilis in swine population: a preliminary investigation

\section{SUMMARY}

Dientamoeba fragilis is a worldwide distributed protozoon which is now estimated responsible of enteric diseases in humans. A wide spectrum of intestinal symptoms has been described: diarrhoea (acute or prolonged), flatulence, abdominal pains, and not specific bowel syndrome; however, asymptomatic infection has been also reported. D. fragilis is still not well know because only the delicate trophozoite stage has been demonstrated in stool samples and definitive identification is based on permanent stains, usually not performed in ova and parasites (O\&P) examinations, in Italy. D. fragilis is difficult to cultivate but molecular techniques, such as PCR, appear promising for a sure diagnosis of this protozoan infection. The epidemiology of dientamoebiasis is not clear too, and this paper presents preliminary results concerning the presence of $D$. fragilis in swine faeces in order to evaluate the possible role of pigs as reservoir of the parasite. So, 121 stool specimens of swine population were examined using Giemsa permanent stain and D. fragilis was observed in 53 stool specimens (43.8\%). Other consideratioona are reported too.

\section{INTRODUZIONE}

Dientamoeba fragilis, protozoo flagellato di recente rivalutazione (5), è attualmente una delle cause più frequenti di infezione intestinale umana protozoaria, quantomeno nei Paesi sviluppati (8), Italia compresa (3). Nell'uomo tale flagellato atipico, di cui non si conosce la fase cistica ma soltanto lo stadio di trofozoite (9), è causa di disturbi intestinali non specifici, di diarrea acuta e protratta, ma può altresì albergare l'intestino umano senza causare danno, quantomeno apparente (2). La individuazione di tale protozoo necessita di un approccio diagnostico tempestivo, data la sua "fragilità" al di fuori dell'ospite abituale (4), che prevede necessariamente colorazioni specifiche permanenti, quali la colorazione di Giemsa o altre, per la sua sicura identificazione (1).

Non si conosce al momento altro ospite al di fuori dell'uomo per $D$. fragilis, sebbene sia stato ipotizzato potersi transitoriamente reperire anche in altri mammiferi, in particolare nel macaco, nel babbuino e nelle pecore $(8,10)$. La delicata e fragile vitalità di tale protozoo non aiuta a comprenderne le vie di trasmissione, sebbene studi recenti sembrano individuare la possibilità di una trasmissione da soggetto a soggetto all'interno di uova di elminti, non soltanto strettamente umani come Enterobius vermicularis, ma anche elminti agenti di zoonosi o comun- que geoelminti come Ascaris spp. e Trichuris spp. $(1,6,9)$.

Da tali premesse è stato impostato uno studio per verificare il ruolo del suino quale potenziale serbatoio di tali protozoi, sulla base della considerazione che tali mammiferi, per certi versi molto affini all'uomo, albergano o possono albergare svariati agenti, batterici e parassitari, responsabili sovente di infezione intestinale umana.

\section{PRESENTAZIONE DELLO STUDIO}

Nel corso del 2006 sono stati raccolti 121 campioni fecali suini, in parte di suini all'ingrasso ed in parte di suini riproduttori; tali campioni sono stati raccolti sia in allevamenti a ciclo chiuso sia in allevamenti a ciclo aperto; parte dei campioni provenivano dal suolo poco dopo la loro eliminazione e parte erano stati prelevati direttamente dall'ampolla rettale. Tutti i campioni sono stati quanto prima analizzati in laboratorio, utilizzando la colorazione permanente di Giemsa (soluzione di Giemsa al $10 \%$ in acqua per 30 minuti) previa fissazione per 1-2 minuti in metanolo (3). La lettura è stata eseguita al microscopio ottico al 100x10 in immersione.

Una parte dei campioni congelata a $-80^{\circ} \mathrm{C}$ per future indagini biomolecolari, sia di conferma che di tipizzazione epidemiologica $(7,11)$.

D. fragilis è stata reperita in 53 campioni fecali, pari al $43.8 \%$ dei casi; essa si presentava come 
trofozoite simil amebico il cui citoplasma si colorava in azzurro (da grigio-azzurro chiaro ad azzurro più intenso), mentre il nucleo si colorava in rosso o rosso-violetto. Solitamente i nuclei erano due, ma talora il nucleo era singolo. In un paio di circostanze i nuclei sembravano essere quattro. Tali nuclei erano frammentati o, comunque, colorati in modo non compatto ed irregolare (Figura 1). Le dimensioni erano comprese tra 5 e $20 \mu \mathrm{m}$ (più spesso tra 7 e $15 \mu \mathrm{m}$ ).

Sono stati osservati anche altri protozoi, ciliati, flagellati ed amebe (soprattutto Iodamoeba buetschlii, l'ameba del maiale, saprofita di tale mammifero), seppure la colorazione di Giemsa non fosse ottimale per la loro identificazione, non sempre infatti avvenuta con certezza (quantomeno in termini di speciazione).

Soltanto in 6 casi $(11.3 \%)$ D. fragilis è stata osservata come protozoo unico, mentre in 57 casi $(88.7 \%)$ era variamente associata ad uno o più protozoi $(8)$.

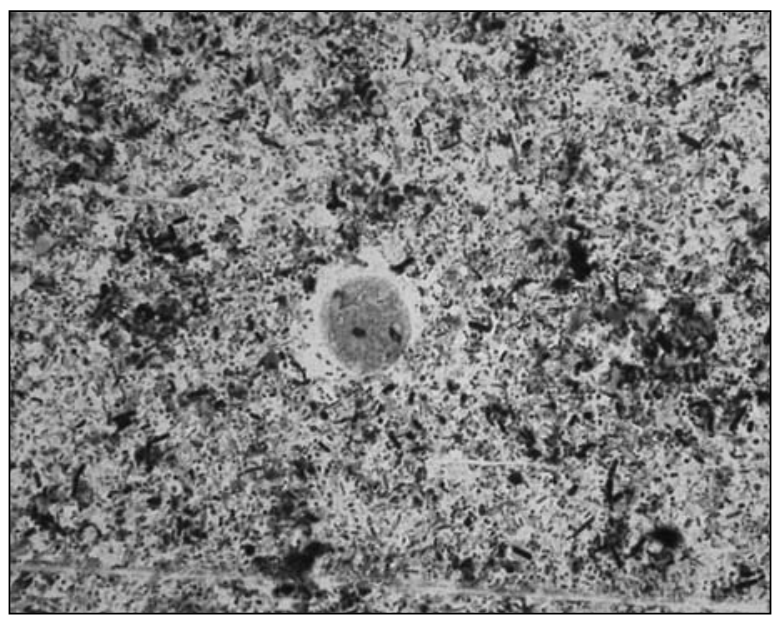

Figura I.

\section{CONCLUSIONI}

D. fragilis, seppure in alcune circostanze di difficile individuazione forse anche perché i modi ed i tempi per la sua colorazione non sono sempre stati pedissequamente rispettati, sembra essere indubbiamente presente: oltre il $40 \%$ dei suini è sembrato infatti essere infettato da questo flagellato, indubbiamente patogeno per l'uomo (mentre, ad esempio, Giardia spp. è stata osservata soltanto in due circostanze, pari all' $1.7 \%$ ). Quanto riportato vuole essere semplicemente una prima fotografia di tale stato di cose; a dire che altre valutazioni dovranno essere fatte per confermare tali dati, soprattutto alla luce della biologia molecolare $(7,11)$. Infatti, dal momento che $D$. fragilis è spesso associata ad altri pro- tozoi non patogeni (presenti sia nello stadio di cisti che di trofozoite), e con questi può presentare analogie morfologiche, è necessaria una certezza diagnostica per discriminare la stessa dagli altri.

L'idea speculativa di questo primo studio era sorta per comprendere il potenziale ruolo di "serbatoio naturale" per tale protozoo da parte del suino. Infatti, dal momento che ancora molto è ignoto circa i meccanismi di trasmissione all'uomo e da uomo a uomo di tale protozoo (1, $6,8,9)$, l'ipotesi di sospettare il suino quale animale serbatoio si poteva fondare su alcune osservazioni, anche personali e recenti. In primo luogo, come introdotto, il fatto che il suino possa spesso albergare nel suo canale intestinale tanti batteri e protozoi (patogeni e non patogeni), responsabili di infezione umana, ci ha spinto a verificare se tra questi non vi potesse essere inclusa anche $D$. fragilis. In secondo luogo abbiamo di recente osservato come in alcune realtà (Perù), in cui la convivenza tra uomo e suino era stretta, non soltanto era presente, nell'uomo, una balantidiasi $\mathrm{o}$, più ancora, una ascaridiasi, ma altresì era vieppiù frequente una dientamoebiasi (dati personali in corso di pubblicazione). Da tutto ciò l'idea di verificare tale stato di cose anche in Italia, e queste prime osservazioni potrebbero confortarci in tale suggestiva ipotesi epidemiologica.

\section{BIBLIOGRAFIA}

1. Bernieri F, Crotti D. Infezioni da protozoi. Microbiol Med 2001; 16: 98-108.

2. Crotti D, D’Annibale ML. Dientamoeba fragilis e dientamoebosi: aspetti di parassitologia clinica e diagnostica di laboratorio. Parassitologia 2001; 43: 135-138.

3. Crotti D, D'Annibale ML, Fonzo G, Lalle M, Cacciò M, Pozio E. Dientamoeba fragilis is more prevalent than Giardia duodenalis in children and adults attending a day care centre in Central Italy. Parasite 2005; 12: $165-170$.

4. De Canale E, Tessari A, Campion L, Rossi L. Dientamoeba fragilis: è realmente fragile? Approccio al trattamento del campione e diagnosi rapida al microscopio. Parassitologia 2003; 45: 1922.

5. Johnson E H, Windsor J J, Graham Clark C. Emerging from Obscurity: Biological, Clinical, and Diagnostic Aspects of Dientamoeba fragilis. CMR 2004; 17: 553-570.

6. Menghi CI, Makiya R, Gatta CL, Méndez OC. Dientamoeba fragilis: molecular biology techniques for the Elucidation of tits mode of transmission. Parassitol Latinoam 2005; 60: 25-31.

7. Stark D, Beebe N, Marriot D, Ellis JT, Harkness J. Detection of Dientamoeba fragilis in fresh stool specimen using PCR. Int J. Parasitol 2005; 35: 57-62.

8. Stark JS, Beebe N, Marriott D, Ellis JT, Harkness J. 
Dientamoebiasis: clinical importance and recent advances. TRENDS in Parasitology 2006; 22: 92-96.

9. Windsor JJ, Rafay AM, Shenoy AK, Johnson EH. Incidence of Dientamoeba fragilis in faecal samples submitted for routine microbiological analysys. Br J Biomed Sc. 1998; 55: 172-175.

10. Windsor JJ, Johnson EH. Dientamoeba fragilis: the unflagellate human flagellate. Br J Biomed Sc 1999; 56: 293-306

11. Windsor JJ, Clark CG, Macfarlene L. Molecular typing of Dientamoeba fragilis. Br J Biomed 2004; Sc. 61: 153-155.

\section{Daniele Crotti}

Strada Comunale per Pilonico Paterno 4 06080 Pianello, Perugia

Tel.: 075602372

E-mail: nenedc@tin.it 